\title{
Richterwahl und Richteramt in einem künftigen Multi- lateralen Investitionsgericht: zentrale Regelungsfragen
}

\author{
Christian Hederer*
}

\section{Zusammenfassung}

Das aktuelle globale Investitionsschutzsystem ist ökonomisch notwendig, aber reformbedürftig. Ein Hauptkritikpunkt am jetzigen Zustand ist die Verfahrensführung durch ad-hoc-Schiedsgerichte, deren Neutralität und Sachkompetenz angezweifelt wird. Deshalb wird u.a. von der Europäischen Union die Etablierung eines ständigen Multilateralen Investitionsgerichts angestrebt. Der Beitrag analysiert zentrale regulatorische Optionen für den Auswahlprozess und die Ausgestaltung der Amtsführung der Richter eines künftigen Multilateralen Investitionsgerichts.

\section{Abstract}

The current global system of investment protection is economically necessary but in need of reform. A major critique of the present system is the conduct of proceedings by ad hoc arbitral tribunals, whose neutrality and competence are contested. This is why the European Union, among others, is advocating the establishment of a standing Multilateral Investment Court. This contribution analyses core regulatory options with respect to the selection and rules of conduct for judges of a future Multilateral Investment Court.

\section{Einleitung und Problemstellung}

In einer globalisierten Wirtschaft sind Schutzmechanismen für grenzüberschreitende Investitionen gegen willkürliche politische oder administrative Eingriffe in den Zielstaaten - insbesondere entschädigungslose Enteignung sowie grob ungerechte und/oder diskriminierende Behandlung - von hoher Bedeutung. In diesem Kontext sind weltweit aktuell etwa 2630 größenteils bilaterale Investitions(schutz)abkommen in Kraft (vgl. UNCTAD 2018a), welche internationalen Investoren die Möglichkeit bieten, Zielstaaten, die solche Eingriffe vornehmen, vor einem internationalen Schiedsgericht auf Schadensersatz zu verklagen. Allein Deutschland hat aktuell 126 derartige Abkommen in Kraft (vgl. Bundesministerium für Wirtschaft und Energie 2018). Die Nutzung dieses völkerrechtlichen Instruments durch Investoren hat sich seit etwa 2003 erheblich intensiviert; pro Jahr werden im Mittel weltweit nunmehr etwa 65 neue Verfahren angestrengt (UNCTAD 2018b).
Parallel zu ihrer steigenden praktischen Bedeutung sind Investitionsabkommen allerdings teils massiver Kritik ausgesetzt (vgl. zusammenfassend Kaufmann-Kohler \& Potestà 2016: 6ff.). Das Kernbedenken besteht in der vermuteten Einschränkung staatlicher Regelungsspielräume. In materieller Hinsicht gründet sich dies auf die zumeist relativ breite und damit erheblichen Interpretationspielräumen unterliegende Formulierung der Investorenrechte: befürchtet wird, dass Staaten bereits im Voraus auf bestimmte Maßnahmen, etwa im Umweltbereich, verzichten könnten, um etwaigen Investorenklagen und in der Folge Schadensersatzzahlungen vorzubeugen. In prozeduraler Hinsicht wird - neben der grundsätzlichen Asymmetrie des Instruments, welches den Investoren das alleinige Klagerecht einräumt - vor allem die Konstituierung und Arbeitsweise der Schiedsgerichte kritisiert. Diese werden für jedes Verfahren ad hoc zusammengestellt und setzen sich regelmäßig aus drei Schiedsrichtern zusammen, von denen jeweils einer durch den klagenden Investor und den klagenden Staat nominiert wird und der dritte - Vorsitzende entweder konsensual oder von einer externen Instanz zu ernennen ist. Die Entscheidung eines Schiedsgerichts ist - abgesehen von eng umschriebenen Annullierungsmöglichkeiten aufgrund grober Rechtsmängel - endgültig. In der Praxis werden entsprechende Verfahren von einer relativ kleinen Elite fast ausschließlich in westlichen Industriestaaten ansässiger Experten betrieben, welche regelmäßig sowohl als Anwälte als auch als Schiedsrichter tätig werden (Langford et al. 2017). Diese Elemente erzeugen Bedenken zur Unabhängigkeit der Schiedsrichter und deren Anreizen, investorenfreundlich zu entscheiden, um neues „Fallgeschäft“ zu generieren (van Harten 2010); zu ihrer Qualifikation (Deutscher Richterbund 2016) und Repräsentativität, zB was überproportional oft beklagte Schwellen- und Entwicklungsländer betrifft (Langford et al. 2017); zur Konsistenz und Vorhersehbarkeit der Urteile angesichts des ad-hocCharakters der Schiedsgerichte und der fehlenden Berufungsmöglichkeit (Kauf- 
mann-Kohler 2016: 6ff.); und schließlich auch zu den Kosten und der Transparenz der Verfahren.

Diese Kritikpunkte werden von der Europäischen Kommission - die seit dem Vertrag von Lissabon für die Verhandlung von Abkommen mit Drittstaaten, welche derartige Investitionsschutzbestimmungen enthalten, zuständig ist - vor allem seit dem Amtsantritt von Handelskommissarin Malmström und auch auf Druck des Europäischen Parlaments und mehrerer Mitgliedstaaten, darunter Deutschland, zunehmend aufgegriffen. Ein Schwerpunkt der Reformbemühungen besteht in der Ersetzung der ad hoc-Schiedsgerichte durch eine ständige Instanz, deren Mitglieder allein durch die vertragsschließenden Staaten ernannt werden. Die auf europäischer Ebene jüngst ratifizierten EU-Handelsabkommen mit Kanada (CETA), Vietnam und Singapur sehen eine solche Instanz auf jeweils bilateraler Ebene bereits vor. ${ }^{1}$ Darüber hinaus engagiert sich die Kommission aber auch für die Einrichtung eines Multilateralen Investitionsgerichts (MIG), das per Beitritt der jeweiligen Vertragsstaaten zu einer entsprechenden Konvention auch für bestehende Abkommen zuständig gemacht werden und damit den herkömmlichen Schiedsgerichtsbarkeitsmechanismus ersetzen könnte. Der EU-Ministerrat hat der Kommission im März 2018 ein Mandat zur Führung entsprechender Verhandlungen im Rahmen der Kommission der Vereinten Nationen für internationales Handelsrecht (UNCITRAL) erteilt. ${ }^{2}$ Der Start konkreter Diskussionen ist für 2019 zu erwarten.

Aus Sicht der Europäischen Kommission würde die Etablierung eines MIG als vollwertiges internationales Gericht, das den Standards bereits etablierter Institutionen, wie etwa des Internationalen Gerichtshofs (IGH), entspricht, die oben skizzierten prozeduralen Probleme des derzeitigen Investitionsschutzsystems im Wesentlichen Iösen (European Commission 2017). Allerdings besteht gegen dieses
Projekt international auch teils erhebliche Skepsis (vgl. z.B. Zuleta 2015). Befürchtet wird unter anderem, dass die derzeit kritisierte vermutete „Investorenfreundlichkeit" der Schiedsrichter durch eine "Staatsfreundlichkeit" von fix durch die Vertragsstaaten ernannten Richtern ersetzt werden könnte, was angesichts des spezifischen Charakters von Investorenklagen besonders problematisch wäre. Zudem ginge die Möglichkeit der Streitparteien, die Auswahl der Schiedsrichter spezifisch auf Gegenstand und Inhalte des jeweiligen Verfahrens abzustimmen, verloren. Generell könnte das Investitionsschutzsystem in höherem Maß politischen Einflüssen unterliegen und damit seinen aus Investorensicht wesentlichsten Vorzug verlieren.

Vor diesem Hintergrund ist zu erwarten, dass der Erfolg eines MIG zentral von seiner konkreten regulatorischen Ausgestaltung und dem Maß, in dem die Anliegen beider Seiten integriert werden können, abhängen wird. Ein neuralgischer Regelungsbereich, der den Fokus dieses Beitrags bildet, ist die Auswahl der Richter und die Ausgestaltung des Richteramtes, etwa in Hinblick auf die Amtsdauer und die Möglichkeit vorzeitiger Abberufung (vgl. auch Kaufmann-Kohler \& Potestà 2017). Die Bedeutung dieser Regeln für die Qualität von Gerichtsurteilen wie auch für die Legitimität des jeweiligen Gerichts als Institution ist sowohl auf nationaler als auch internationaler Ebene allgemein anerkannt. Die Analyse zentraler regulatorischer Optionen für ein MIG ist diesem Bereich ist Gegenstand dieses Beitrags.

Der Beitrag gliedert sich wie folgt. Abschnitt 2 skizziert einen analytischen Beurteilungsrahmen in Hinblick auf den Charakter eines MIG als internationales Gericht. Zunächst werden zentrale Anforderungen an einen solches Gericht als Institution, und aufbauend darauf, an seine Richter diskutiert. In Anschluss daran wird gezeigt, dass manche dieser Anforderungen Zielkonflikten unterliegen, die aktive regulatorische Entschei- dungen notwendig machen. Auf dieser Grundlage diskutiert Abschnitt 3 dann konkrete regulatorische Optionen für Richterwahl und richterliche Amtsführung in einem MIG. Dies erfolgt in einer vergleichenden Perspektive unter Analyse von Regelungen und Erfahrungen ausgewählter bereits etablierter internationaler Gerichte, aber auch unter Berücksichtigung der skizzierten spezifischen Charakteristika und Bedürfnisse des internationalen Investitionsschutzes. Ein kurzes Fazit schließt den Beitrag.

\section{Beurteilungskriterien internationaler Gerichte \\ und ihrer Richter}

\subsection{Kriterien}

Analog ihren nationalen Pendants sind internationale Gerichte in grundsätzlicher Betrachtung rechtsstaatliche Institutionen, d.h. sie dienen der Lösung von Konflikten auf Grundlage völkerrechtlich verbindlicher Normen und beugen damit auch einer willkürlichen Machtausübung größerer gegen kleinere Staaten vor (Chesterman 2008). Dies wirft die Frage auf, warum souveräne Staaten überhaupt zu einer Abtretung entsprechender Kompetenzen an internationale Gerichte bereit sind. In der aktuellen Literatur gibt es dazu verschiedene, letztlich aber komplementäre Antworten (vgl. Posner \& Yoo 2005, Helfer \& Slaughter 2005, Voeten 2013). Zentral ist die Schaffung einer Vermittlungsinstanz, die von den partizipierenden Staaten als neutral betrachtet wird. Diese Neutralität erleichtert die a-priori-Betrauung des Gerichts mit Konflikten, die zum Zeitpunkt des Abschlusses eines völkerrechtlichen Vertrags in keinem Fall vollständig vorhergesehen werden können, und erhöht die Wahrscheinlichkeit, dass die der Lösung eines Konflikts zugrunde liegende Interpretation von Fakten und Rechtsgrundlagen durch das Gericht die Zustimmung aller Parteien findet. Zudem kann die Unterwerfung unter die Rechtsprechung eines internationalen Gerichts gegenüber anderen

1 Comprehensive Economic and Trade Agreement (CETA) Art 8.29, http://trade.ec.europa.eu/doclib/docs/2014/september/tradoc_152806.pdf; EU-Vietnam Free Trade Agreement Chapter 8/2, Section 3 Art 15, http://trade.ec.europa.eu/doclib/press/index.cfm?id=1437; EU-Singapore Investment Protection Agreement, Art 3.9, http://trade.ec.europa.eu/doclib/press/index. $\mathrm{cfm}$ ?id=961 (alle zuletzt abgerufen am 01.03.2019).

2 Council of the European Union (2018) Negotiating directives for a Convention establishing a multilateral court for the settlement of investment disputes, Brüssel. http://data.consilium. europa.eu/doc/document/ST-12981-2017-ADD-1-DCL-1/en/pdf (zuletzt abgerufen am 01.03.2019). 
Staaten und Akteuren als Signal für die Bereitschaft eingesetzt werden, die eingegangenen völkerrechtlichen Verpflichtungen tatsächlich einzuhalten - was gerade im Investitions- und Handelsbereich mit positiven ökonomischen Konsequenzen verbunden sein kann.

Aus diesen Überlegungen ergeben sich mehrere Kriterien, anhand derer die Qualität eines internationalen Gerichts grundsätzlich beurteilt werden kann. Erstens sollte das Gericht als Institution unabhängig sein, d.h. gegenüber allen relevanten Akteuren einen hohen Grad an Autonomie genießen, insbesondere auch in budgetärer und administrativer Hinsicht (vgl. z.B. Brown 2003, Mahoney 2008). Zweitens sollte das Gericht unparteiisch sein, was auch eine hohe juristische Qualität seiner Entscheidungen impliziert (z.B. bezüglich Qualität und Detail der seinem Urteil zugrundeliegenden Argumente, oder der Konsistenz der Urteile). Drittens sollte das Gericht ein hohes Maß an Legitimität genießen. Im allgemeinsten Sinn bedeutet dies eine grundsätzliche Akzeptanz seiner Rechtsprechungsfunktion und Urteile durch den betroffenen Adressatenkreis; im Regelfall wird diese Akzeptanz eng an die oben genannten Kriterien Unparteilichkeit, Unabhängigkeit und Qualität geknüpft sein (vgl. Grossman 2009, Cohen et al. 2018). Schließlich sollte das Gericht auch ein Mindestmaß an faktischer Wirksamkeit aufweisen; das heißt vor allem, dass Staaten sich des Gerichts zur Konfliktlösung auch tatsächlich bedienen und seine Urteile effektiv umsetzen (Posner \& Yoo 2005: 28f.).

Aus diesen Kriterien für das Gericht als Institution können nun weitere Kriterien für die - hier in erster Linie zur Diskussion stehenden - Richter als Gruppe von Individuen abgeleitet werden, ${ }^{3}$ die in Abschnitt 3. auch der Analyse der Optionen für ein MIG zugrundegelegt werden. Zentral ist zunächst wiederum die richterliche Unabhängigkeit, also ein hohes Maß an Autonomie auch gegenüber dem Gericht selbst und das Fehlen persönlicher Interes- sen oder sonstiger Faktoren, die einer rein rechtsfokussierten Fallbeurteilung entgegenstehen. Das Kriterium der Unparteilichkeit impliziert auf individueller Ebene auch ein hohes Maß an persönlicher Integrität und Professionalität bis hin zur Erfüllung allgemeiner charakterlicher Anforderungen. Des weiteren müssen alle Richter bestimmte Qualifikationsanforderungen erfüllen, die als (formale) Befähigung zu nationalen Richterämtern, aber auch (inhaltliche) Anforderungen bez. Sachkompetenz und/oder (Berufs-)Erfahrung formuliert sein können. Schließlich bildet gerade für die Richterschaft internationaler Gerichte auch deren Repräsentativität ein wichtiges Kriterium. Diese richtet sich traditionell nach verschiedenen geografischen Kriterien, schließt aber zunehmend auch das Geschlecht der Richter/innen mit ein.

\subsection{Zielkonflikte}

Auf den ersten Blick kann eine bestmögliche Erfüllung dieser Kriterien auch für die regulatorische Ausgestaltung eines MIG als "Goldstandard" betrachtet werden. Allerdings bestehen zwischen einzelnen der Kriterien Zielkonflikte, die, wie im folgenden Abschnitt gezeigt wird, auch für den spezifischen Kontext des Investitionsschutzsystems teilweise von hoher Bedeutung sind.

Richterliche Unabhängigkeit und Unparteilichkeit werden zumeist als komplementär bzw wechselseitig verstärkend angesehen. Sie können im Kontext von Verhaltens- und Abberufungsregeln für das Richteramt aber auch in einem Spannungsverhältnis stehen: wenn beispielsweise die vorzeitige Abberufung eines Richters sehr strengen Voraussetzungen unterliegt, stärkt dies zwar dessen Unabhängigkeit, reduziert aber gleichzeitig seine Kontrollierbarkeit in Hinblick auf die Einhaltung der genannten Standards während der Amtsführung. Verbunden damit ist das kontroverse Verhältnis zwischen der Unabhängigkeit des Gerichts und seiner Richter und seiner faktischen Wirksamkeit. Die konventionelle Betrachtungsweise geht hier wie- derum von einem weitgehend komplementären Verhältnis aus. Posner und Yoo (2005) haben in einem einflussreichen Beitrag jedoch argumentiert, dass ein hohes Maß an richterlicher Unabhängigkeit den faktischen Mehrwert des Gerichts für die Vertragsstaaten reduzieren kann, weil Freiräume für eine Rechtsprechung geschaffen werden, die persönlichen, oft auch ideologisch motivierten Präferenzen der Richter mehr entspricht als den Interessen der Konfliktparteien - ein Zielkonflikt, der letztlich zur Notwendigkeit der Festlegung eines „politisch optimalen“ Maßes an Unabhängigkeit führen würde (Voeten 2013: 424).

Ein weiteres Spannungsfeld besteht zwischen Repräsentativität und Unabhängigkeit / Unparteilichkeit. Dies deshalb, weil ein striktes Verständnis der letzteren beiden Prinzipien die Entscheidung des Richters eigentlich vollständig von dessen persönlichem Hintergrund ablösen sollte: ein "home state bias" richterlicher Argumentation ist ausdrücklich unerwünscht (Voeten 2014). Zielkonflikte können auch zwischen Repräsentativität und Qualifikation und im weiteren auch der juristischen Qualität der Gerichtsurteile bestehen. Beispielsweise sind Prozesse der Richterauswahl, die auf eine bestmögliche geografische Repräsentation abzielen, oft hochgradig politisiert und führen damit regelmäßig nicht zur Wahl der bestqualifizierten Kandidaten. Andererseits können sehr strikte Qualifikationsanforderungen ein gewünschtes Maß an Repräsentativität gefährden, insofern unterrepräsentierte Gruppen (z.B. kleinere Entwicklungsländer) zu wenige adäquate Kandidaten stellen können. Schließlich kann eine sehr heterogene Richterschaft zwar modernen Repräsentationsanforderungen besser entsprechen, andererseits aber die Konsensfindung und damit auch die faktische Wirksamkeit des Gerichts beeinträchtigen.

\footnotetext{
3 Vgl. für internationale (wenngleich unverbindliche) einschlägige Richtlinien die „Burgh House Principles of the International Judiciary“ (2004) der International Law Association Study Group on the Practice and Procedure of International Tribunals, https://www.ucl.ac.uk/international-courts/sites/international-courts/files/burgh_final_21204.pdf; "Resolution on the Values Pertaining to Judicial Appointments to International Courts and Tribunals" (2011) des International Bar Associations's Human Rights Institute, https://www.ibanet.org/Human_Rights_Institute/ council-resolutions.aspx; und die "Resolution on the Position of the International Judge (2011) $6^{\text {th }}$ Commission of the International Institute of International Law, http://www.idi-iil.org/app/ uploads/2017/06/Question1.pdf (alle zuletzt abgerufen am 01.03.2019).
} 


\section{Optionen für ein Multilaterales Investitionsgericht}

Auf Grundlage der obigen Diskussion kann nunmehr eine vergleichende Analyse regulatorischer Optionen vorgenommen werden, wobei grundsätzlich die Phase der Richterwahl bzw -ernennung (3.1 und 3.2) von jener der Amtsführung (3.3. und 3.4) unterschieden wird. Für jeden Aspekt erfolgt zunächst ein kurzer Überblick bestehender Regelungen in den folgenden internationalen Gerichten: IGH; Internationaler Strafgerichtshof (IStGH); Internationaler Seegerichtshof (ISGH); WTO-Berufungsgremium (WTO BG, hier vereinfachend als ebenfalls als "Gericht" eingestuft); Europäischer Gerichtshof (EuGH); und Europäischer Gerichtshof für Menschenrechte (EGMR). ${ }^{4}$ Die Analyse geht davon aus, dass die materiellen Schutzstandards bestehender Investitionsschutzverträge im wesentlichen unverändert bleiben, die analysierte hypothetische Reform sich also auf verfahrensrechtliche Aspekte beschränkt. Auf die mögliche Einrichtung einer Berufungsinstanz und etwaige gesonderte Kriterien für eine solche kann aus Raumgründen nicht eingegangen werden; ebenso müssen die für die Amtsführungsphase getroffenen Regelungen der Zuteilung der Richter zu bestimmten Fällen und einer möglichen Suspendierung von denselben unberücksichtigt bleiben.

\subsection{Auswahlanforderungen}

a) Bestehende Regelungen.

Bezüglich der Unabhängigkeit von Kandidaten formulieren die betrachteten Gerichte mit Ausnahme des EuGH keine expliziten Anforderungen; Anforderungen für die persönliche Integrität sind zumeist recht allgemein gehalten (die gängigste Formulierung rekurriert auf einen „high moral character“). Für diese Kriterien liegt der Regelungsschwerpunkt in der Amtsführungsphase (vgl. unten 3.4).

Was die Qualifikationsanforderungen betrifft, findet sich ein reines Abstellen auf Expertise und Erfahrung (ISGH, WTO BG) ebenso wie eine Alternative zwischen "anerkannter Kompetenz" und einer formalen Befähigung für das nationale Richteramt (IGH, EuGH, EGMR). Der IStGH bildet einen Sonderfall: einerseits wird die verlangte Expertise in zwei Kategorien (Strafund Völkerrecht) unterteilt, wobei die Richter beider „Expertengruppen“ einen gewissen Mindestanteil nicht unterschreiten dürfen. Andererseits wird bezüglich der formalen Qualifikation verbindlich eine Befähigung zu höchsten nationalen Richterämtern verlangt.

Bezüglich der Repräsentativität ist zunächst zu differenzieren, ob die Zahl der vorgesehenen Richter jener der Vertragsstaaten entspricht ("volle“ Repräsentation; hier EuGH und EGMR) oder diese unterschreitet („selektive“ Repräsentation; IGH, IStGH, ISGH, WTO BG) (vgl. Voeten 2009). Während die geografische Repräsentation in der ersteren Gruppe unproblematisch ist, bestehen in der letzteren durchgehend explizite Regelungen, die vor allem auf Repräsentation betimmter Regionen oder Rechtstraditionen abstellen und tendenziell desto detaillierter ausfallen, je höher die Zahl der Richter im Verhältnis zur Zahl der Vertragsstaaten ist. Bez. des Anteils von Männern und Frauen bestehen für keines der betrachteten Gerichte verbindliche Anforderungen; für den IStGH und informell auch für den EGMR bestehen allerdings explizite Empfehlungen bzw. Bestrebungen im Auswahlprozess, den Frauenanteil in der Richterschaft zu erhöhen. b) Überlegungen für das MIG.

Eine Befolgung der skizzierten Regelungspraxis bez. Unabhängigkeit und Integrität erscheint für das MIG unproblematisch. Bez. Qualifikation sind angesichts der skizzierten Kritikpunkte am bestehenden System prinzipiell hohe Standards zu empfehlen, die allerdings so ausbalanciert werden sollten, dass das Richteramt zumindest längerfristig nicht nur der jetzt dominierenden Expertenelite zugänglich ist, sondern angemessenen Repräsentativitätsanforderungen genügen kann (siehe unten). Dies impliziert, dass die formale Qualifikationsanforderung des IStGH, die augenscheinlich am spezifischen Charakter dieses Gerichts als strafrechtliche Institution orientiert ist, für ein MIG wohl zu weit gehen würde. Die IStGHRegelung zu den Expertengruppen ist hingegen für ein MIG sehr interessant. Investitionsschutzverfahren erfordern Expertise zumeist nicht nur in internationalem Investitionsrecht im engeren Sinne, sondern sehr oft auch in internationalem Handelsrecht, Völkerrecht und spezifischeren Rechtsmaterien (auf internationaler wie nationaler Ebene) wie Umweltrecht, Verwaltungsrecht oder Grund- und Menschenrechte. Eine Unterteilung der Qualifikationsanforderungen in Gruppen könnte (neben anderen Maßnahmen wie der Nominierung von ad hoc-Richtern oder Experten für bestimmte Fälle) diesem Spezifikum besser gerecht werden als das jetzige System, wobei auch hier der erwähnte Zielkonflikt mit den Repräsentativitätsanforderungen im Auge zu behalten ist.

Bez. Repräsentativität ist zunächst wiederum zu unterscheiden, ob volle oder selektive Repräsentation vorgesehen ist. Dies wird klarerweise primär von der Anzahl der Vertragsstaaten des MIG abhängen, wobei bez. der Entscheidung über die Zahl der Richter ei-

\footnotetext{
4 Die im folgenden angeführten Einzelregelungen werden aus Raumgründen nicht im Detail referenziert. Rechtsquellen für die genannten Gerichte sind folgende: IGH: Statute of the International Court of Justice, 24 October 1945; Rules of Court, International Court of Justice, adopted on 14 April 1978. IStGH: Rome Statute of the International Criminal Court, doc. A/CONF.183/9 of 17 July 1998; entered into force on 1 July 2002; Rules of Procedure and Evidence, Official Recordings of the Assembly of States Parties to the Rome Statute of the International Criminal Court, First Session, New York, 3-10 September 2002 (ICC-ASP/1/3 and part II.A. including subsequent Amendments (International Criminal Court, 2005); Code of Judicial Ethics, International Criminal Court ICC-BD/02-01-05. ISGH: United Nations Convention on the Law of the Sea, adopted 10 December 1982; Statute of the International Tribunal for the Law of the Sea, Annex VI to UN Convention on the Law of the Sea; Rules of the Tribunal, International Tribunal for the Law of the Sea ITLOS/8, 17 March 2009. WTO BG: Understanding on Rules and Procedures Governing the Settlement of Disputes, Annex 2 of the Agreement Establishing the World Trade Organization; Working Procedures for Appellate Review, WTO WT/AB/WP/6, 26 August 2010 (including Annex II, Rules of Conduct for the Understanding of Rules and Procedures Governing the Settlement of Disputes). EuGH: Treaty of the Functioning of the European Union, Offical Journal C 326, 26/10/2012P, 1-390; Statute of the Court of Justice of the European Union, Protocol no 3, consolidated version; Rules of Procedure of the Court of Justice, 25 September 2012, including subsequent Amendments. EGMR: Convention for the Protection of Human Rights and Fundamental Freedoms, Rome, 4.XI.1950 (as amended by Protocols Nos 11 and 14; supplemented by Protocols Nos 1, 4, 6, 7, 12 and 13); Resolution on Judicial Ethics, Council of Europe, adopted by the Plenary Court on 23 June 2008; Rules of Court, European Court of Human Rights, 14 November 2016.
} 
nes MIG auch der Zielkonflikt zwischen Heterogenität und Kohäsion bzw. der Fähigkeit einer konsensualen Urteilsfindung im Auge zu behalten ist. Im Fall voller Repräsentation könnte die relativ unproblematische Umsetzung geografischer Anforderungen für eine Verbesserung der Erfüllung anderer Kriterien genutzt werden, indem jeder Staat zu einer Nominierung mehrerer Kandidaten (optional auch mit fremder Nationalität, um die Adressierung von möglichen personellen Engpässen bestimmter Vertragsstaaten zu erleichtern) verpflichtet wird, was etwa die Erreichung eines bestimmten Frauenanteils, aber auch eines differenzierteren Qualifikationsprofils der Richterschaft (siehe oben) erleichtern sollte. Im Fall selektiver Repräsentation wäre insbesondere eine Anforderung bez. Vertretung verschiedener Rechtskreise auch fachlich sinnvoll. Darüber hinaus könnten aber auch investitionsspezifische Aspekte berücksichtigt werden, etwa indem führende Herkunfts- oder Zielstaaten internationaler Investitionen ständig repräsentiert sind oder Vertretungen bestimmter Ländergruppen, die nach ihrem ökonomischen Entwicklungsstand differenziert werden, geschaffen werden. Der angesprochene Zielkonflikt zur konsensualen Urteilsfindung würde sich allerdings bei letzterer Variante mit besonderer Schärfe stellen.

\subsection{Auswahlverfahren}

Die Gestaltung des Auswahlverfahrens für die Richter eines MIG ist nicht nur für die effektive Umsetzung der oben genannten Auswahlkriterien von zentraler Bedeutung, sondern auch für die Legitimität und faktische Wirksamkeit des Gerichts. Prinzipiell gliedert sich das Verfahren in die Nominierung von Kandidaten auf nationaler Ebene und den anschließenden internationalen Prozess, der entweder in einer sofortigen Wahl durch die Vertreter der partizipierenden Staaten (mit variierenden Mehrheitserfordernissen) oder einem weiteren "screening" der Kandidaten, etwa durch ein dazu beauftragtes Expertenkomitee, bestehen kann (vgl. Kohler-Kaufmann \& Potestà 2017: $60 \mathrm{ff})$. a) Bestehende Regelungen.

Für die Modalitäten bzw den Prozess der Nominierung auf nationaler Ebene existieren generell keine verbindlichen Regelungen. Was die Zahl der zu nominierenden Kandidaten betrifft, legt der EGMR eine Verpflichtung von drei Personen fest. IGH und ISGH erlauben eine Nominierung von maximal vier bzw zwei Personen. Ansonsten bildet die Nominierung von einem Kandidaten pro Staat den Regelfall.

Auf internationaler Ebene sieht nur der ISGH eine Wahl der Richter unmittelbar nach der nationalen Nominierung vor. Beim IGH und optional auch beim IStGH obliegt die formale Nominierung der Kandidaten auf internationaler Ebene der jeweiligen Gruppe nationaler Vertreter beim Ständigen Schiedshof in Den Haag, wobei die Autonomie dieser Gruppe, von den Vorschlägen ihrer Heimstaaten abzuweichen, von Land zu Land variiert. Beim WTO BG, EuGH, EGMR und wiederum optional beim IStGH sind - teilweise auf informeller Ebene - Komitees eingerichtet, die insbesondere die Erfüllung der Anforderungen an Qualifikation und Integrität durch die nationalen Kandidaten sicherstellen sollen.

Hintergrund der Einrichtung dieser Komitees war die oft beklagte Politisierung des Auswahlprozesses von Richtern internationaler Gerichte, die insbesondere für den IGH und den IStGH relativ gut dokumentiert ist (MacKenzie 2010: Kap. 4). Der Richterwahl und -ernennung in diesen Institutionen gehen regelmäßig intensive Kampagnen, Verhandlungen und politische „Kuhhändel" voraus, die oft in einem breiteren Kontext der Verteilung von Macht und Einfluss in internationalen Organisationen angesiedelt sind. Die Vermutung liegt nahe - wenn auch diesbezügliche "harte" empirische Evidenz natürlich nur schwer zu gewinnen ist - dass derartige Arrangements die Qualität der gewählten Richter, und damit auch die Legitimität und faktische Wirksamkeit der Gerichte, teilweise empfindlich beeinträchtigen (vgl. für den EGMR Kosař 2015). Angesichts dieses politischen Charakters variiert der faktische Einfluss der Komitees stark und ist auch fraglich, inwieweit die Auswahl der
Komiteemitglieder selbst wiederum politischen Einflüssen unterworfen ist.

Eine wichtige Variable auch für die politische Komponente der Richterwahl sind schließlich die Mehrheitserfordernisse. Hier ergibt sich bei den betrachteten Gerichten ein sehr heterogenes Bild, das hier nur grob wiedergegeben werden kann. EuGH und WTO BG erfordern im Wesentlichen eine einstimmige Entscheidung der Staatsregierungen. ISGH und IStGH erfordern eine Zweitdrittelmehrheit, der EGMR eine einfache Mehrheit der wahlberechtigten Versammlung von Staatsvertretern; im einzelnen sind hier die Wahlprozesse bis zur Besetzung aller Positionen komplex - auch weil z. T. pro Staat mehr als ein Kandidat nominiert wird, vgl. oben - und können mehrere Durchgänge erfordern. Eine Wahl zum Richter des IGH erfordert schließlich eine einfache Mehrheit sowohl in der UN-Vollversammlung als auch im UNSicherheitsrat.

\section{b) Überlegungen für das MIG.}

In Hinblick auf die Auswahl der Richter eines MIG ist zunächst festzuhalten, dass ein gewisses Maß an „Politisierung" des Prozesses angesichts der geforderten Abgabe von Souveränität der Vertragsstaaten an das Gericht unvermeidlich ist. Andererseits zählt die Möglichkeit eines politisch beeinflussten Gerichts, wie erwähnt, zu den Hauptbefürchtungen der Skeptiker eines MIG, und sollten daher regulatorische Möglichkeiten einer Versachlichung des Auswahlprozesses, wie sie von anderen Gerichten vorgezeichnet werden, jedenfalls genutzt werden.

Daraus ergibt sich zunächst, dass die bestehende Praxis, abgesehen von einer Regelung zur Zahl der Kandidaten pro Staat nicht in nationale Nominierungsprozesse einzugreifen, auch für das MIG sinnvoll erscheint. Wie bereits in Abschnitt 3.1 erwähnt, erscheint die Schaffung einer Möglichkeit oder sogar Verpflichtung für jeden Vertragsstaat, mehr als einen Kandidaten zu nominieren, für ein MIG sowohl aus Qualifikations- als auch Repräsentationsgesichtspunkten empfehlenswert. Allerdings führt eine größere Zahl von Kandidaten zu einer höheren Komplexität, und 
damit auch potenziell höheren Politisierung, des Wahlprozesses. Dem könnte mit der Einrichtung eines Expertenkomitees nach Vorbild insbesondere des EuGH und des EGMR begegnet werden. Ein solches Komitee könnte auch nicht-staatliche Vertreter - insbesondere internationaler Investoren, aber auch involvierter NGOs - zumindest in einer beratenden Rolle mit einschließen und/oder um eine umfassende Stakeholder-Konsultation, wie sie insbesondere auf europäischer Ebene immer üblicher wird, ergänzt werden. Auf diese Weise könnte die Legitimität des MIG insgesamt erhöht und auch den bestehenden Bedenken von Investorenseite bezüglich der Unparteilichkeit seiner Richter begegnet werden. Die Arbeiten des Komitees würden dann in eine begründete Empfehlung an das wahlberechtigte Gremium münden.

Regulatorische Entscheidungen zur Zusammensetzung des Wahlgremiums und zu den Mehrheitserfordernissen hängen sehr stark von der Zahl der Vertragsstaaten des MIG ab. Bei einem kleineren und relativ homogenen Kreis erscheint nicht zuletzt aus Legitimitätsgründen ein Einstimmigkeitserfordernis durchaus sinnvoll. Allerdings sollte den potenziellen Problemen infolge von Beitritten weiterer Staaten nach Möglichkeit bereits vorab durch Festlegung gewisser Übergangsregelungen, beispielsweise in Richtung einer Abschwächung der Mehrheitserfordernisse, vorgebeugt werden. Einzelne Autoren (z.B. Kaufmann-Kohler \& Potestà 2016: 60) schlagen vor, die Richterwahl für ein MIG von vorneherein in der UN-Vollversammlung anzusiedeln. Dies erscheint aber nicht zuletzt angesichts der Erfahrungen mit der Politisierung des IGH-Auswahlprozesses (siehe oben) wenig sinnvoll.

\subsection{Amtsdauer}

Bez. Amtsdauer sind prinzipiell deren Länge und Erneuerbarkeit, d.h. die Möglichkeit einer Wiederwahl, zu regeln. Zumeist wird davon ausgegangen, dass eine lange Amtsdauer und der Ausschluss einer Wiederwahl die Unabhängigkeit und Unparteilichkeit der Richter sowie die Qualität der Gerichtsurteile erhöhen, weil die Richter sich besser in ihre Materie einarbeiten, einen „ésprit de corps“ entwickeln und weitgehend unabhängig von künftigen Karrieremotiven entscheiden können. Allerdings stellt sich die Frage des Zielkonflikts mit der faktischen Wirksamkeit des Gerichts (siehe oben 2.2.).

a) Bestehende Regelungen.

Dem Standard langer und nicht erneuerbarer Amtsdauern wird durch den IStGH und den EGMR (jeweils 9 Jahre) entsprochen. Die Amtsdauern der anderen betrachteten Gerichte sind erneuerbar und reichen von 9 Jahren (IGH und ISGH) bis zu 4 Jahren (WTO BG). Der generelle Trend geht in Richtung einer Verlängerung der Amtsdauern; so wurde die Amtsdauer der Richter des EGMR 2010 von 6 auf 9 Jahre erhöht, ähnliche Projekte existieren auch für den WTO BG (vgl. Steger 2015: 463).

b) Überlegungen für das MIG.

Auf den ersten Blick erscheint eine lange Amtsdauer für die Richter eines MIG vor allem auch angesichts der Kritik an der Qualität und Konsistenz der schiedsgerichtlichen Urteile im jetzigen System (vgl. oben 1.) empfehlenswert. Dem stehen die skizzierten Befürchtungen bez. der faktischen Wirksamkeit des Gerichts gegenüber. Diese müssen allerdings in den spezifischen Kontext der Investionsgerichtsbarkeit gestellt werden, in der nur Investoren, nicht aber Staaten klagsberechtigt sind. Vor diesem Hintergrund kann ein hohes $\mathrm{Maß}$ an richterlicher Unabhängigkeit zwei Effekte generieren: einerseits könnten Richter, die weitgehend losgelöst von den Interessen der Vertragsstaaten agieren, die Klagserhebung für Investoren im Vergleich zu einer Konstellation mit einem geringeren $\mathrm{Ma}$ an Unabhängigkeit attraktiver machen und damit die Wirksamkeit des Systems, gemessen an seinem Nutzungsgrad, sogar erhöhen. Andererseits könnten sich Staaten angesichts dieser Situation schrittweise aus dem System zurückziehen und/oder die Qualität der Urteilsumsetzung verschlechtern. Im Lichte dieser Ambivalenz empfiehlt es sich, den Regelungsschwerpunkt für ein MIG eher auf die Sicherung der richterlichen Unparteilichkeit im Rahmen von Amtsführungsstandards zu legen (siehe unten) und bez. der Amtsdauer im Spektrum der bestehenden Regelungen einen Mittelweg einzuschlagen. Sinnvoll wäre damit etwa eine nicht erneuerbare Amtsdauer von ca. 6 Jahren.

\subsection{Amtsführungsstandards und Abberufungsgründe}

a) Bestehende Regelungen.

Die Amtsführungsstandards in den betrachteten Gerichten beziehen sich in erster Linie auf der Sicherstellung die richterlichen Unabhängigkeit und Unparteilichkeit. Dies erfolgt über mehrere Arten von Anforderungen. Erstens bestehen Regelungen bezüglich möglicher anderer Beschäftigungen. Ein Richteramt am IGH, EuGH, EMGR und mit Einschränkungen auch am IStGH kann nur als Vollzeitberuf ausgeübt werden; ISGH und WTO BG erfordern zumindest eine ständige Verfügbarkeit. Zweitens schließen alle Gerichte die parallele Bekleidung eines politischen oder administrativen Amtes sowie jede zusätzliche juristische Berufsaktivität aus. Diese Unvereinbarkeitsregeln werden am IStGH und EGMR auf die Vermeidung des bloßen Anscheins eines Interessenskonflikts ausgeweitet; beim WTO BG sind für alle Aktivitäten, die Zweifel an der Unabhängigkeit oder Unparteilichkeit erwecken könnten, strikte Offenlegungspflichten normiert. IStGH und EGMR schreiben darüber hinaus ausdrücklich ein richterliches Verhalten vor, welches die Reputation des Gerichts und das in dieses gesetzte öffentliche Vertrauen nicht beeinträchtigt. Drittens finden sich beim WTO BG, EGMR und EuGH auch Einschränkungen, was die berufliche Tätigkeit nach Verlassen des Richteramts betrifft, wobei erstere beiden Gerichte eine anwaltliche Rolle vor dem Gericht für eine bestimmte Periode ausdrücklich ausschließen.

Die Gründe für eine vorzeitige Abberufung aus dem Richteramt beschränken sich bei der Mehrzahl der betrachteten Gerichte auf die Formulierung, dass der Betroffene die Voraussetzungen für die Ausübung seines Amtes nicht mehr erfülle. In prozeduraler Hinsicht wird die Entscheidung zumeist dem Gericht selbst überlassen, wobei 
die Mehrheitserfordernisse variieren (Einstimmigkeit der Richter bei IGH, EuGH und ISGH; Zweidrittelmehrheit bei EGMR, jeweils unter Ausschluss des Betroffenen). Der IStGH bildet in beiden Punkten eine Ausnahme, weil einerseits die Abberufungsgründe wesentlich detaillierter definiert und unterschiedliche "Schweregrade" des Fehlverhaltens definiert werden und andererseits ein externes Gremium, nämlich die UN-Vollversammlung, mit der Entscheidung über eine Abberufung betraut wird. Der WTO BG weist keine expliziten Regelungen zu einer Abberufung auf.

b) Überlegungen für das MIG.

Vor dem Hintergrund der skizzierten Kritik am gegenwärtigen System, insbesondere der Struktur der einschlägigen Expertentätigkeit ( $\mathrm{vgl}$ oben I.) und den erwähnten Bedenken bez. der faktischen Wirksamkeit des MIG sollten die Amtsführungsstandards grundsätzlich streng formuliert und mit einer realistischen Abberufungsmöglichkeit verbunden werden. Im Spektrum der bestehenden Regelungen bedeutet dies, dass strikte Regelungen zu Unvereinbarkeit und Interessenskonflikten aufgestellt werden sollten, welche auch die Anscheinsvermeidung mit einschließen. Eine parallele Aktivität als Richter und Anwalt wäre damit für die Dauer der Amtszeit jedenfalls ausgeschlossen. Dem gleichen Zweck würden Einschränkungen für die Zeit nach der Amtsausübung nach dem Vorbild von WTO BG, EuGH oder EGMR dienen. Allerdings ist auch darauf zu achten, dass diese Einschränkungen zumindest in der Anfangsperiode des MIG nicht zu restriktiv formuliert werden, um die Attraktivität des Richteramts für qualifizierte Kandidaten nicht über Gebühr einzuschränken. Was die Abberufungsgründe betrifft, wäre in prozeduraler Hinsicht jedenfalls einer Lösung der Vorzug zu geben, die den partizipierenden Staaten ein institutionelles Mitspracherecht einräumt.

\section{Fazit}

Das internationale Investitionsschutzsystem steht an einem Scheideweg. Der grundsätzlichen Notwendigkeit von Schutzmechanismen für internati- onale Investoren in einer globalisierten Wirtschaft steht ein inzwischen weithin anerkannter Reformbedarf gegenüber. Die Etablierung eines Multilateralen Investitionsgerichts hat grundsätzlich das Potenzial, das System auf eine tragfähigere Grundlage zu stellen; seine tatsächliche Einrichtung und sein Erfolg werden aber wesentlich von seiner konkreten regulatorischen Ausgestaltung abhängen. Dieser Beitrag beschäftigte sich mit einem Kernbereich eines künftigen $\mathrm{MIG}$, nämlich der Auswahl und Amtsführung seiner Richter. Unbeschadet einer Analyse der konkreten politischen Realisierungsschancen eines MIG, die in diesem Rahmen nicht geleistet werden konnte, wurden mehrere Regulierungsprobleme identifiziert, deren Adressierung wesentlich zum Erfolg oder Misserfolg der neuen Institution beitragen könnte. Es sind dies insbesondere die Findung einer adäquaten Balance zwischen Qualifikations- und Repräsentationsanforderungen, die Gestaltung des Auswahlprozesses im Spannungsfeld politischer und fachlicher Notwendigkeiten sowie eine Kalibrierung der Regeln für die richterliche Amtsführung und vorzeitige Abberufung, welche die klassischen Anforderungen an Unabhängigkeit und Unparteilichkeit ebenso berücksichtigt wie die Interessen der souveränen Staaten, die sich der Gerichtsbarkeit des MIG freiwillig unterwerfen. Wie gezeigt wurde, kann die Identifizierung von Lösungsansätzen für diese Probleme wesentlich von einer vergleichenden Betrachtung mit anderen internationalen Gerichten profitieren. In dieser Sicht könnte ein MIG letztlich zum integralen Bestandteil eines größeren Systems werden, das zu einer Stärkung rechtsstaatlicher Prinzipien auch auf internationaler Ebene beiträgt. 


\section{LITERATUR}

Bundesministerium für Wirtschaft und Energie (2018) Übersicht über die bilateralen Investitionsförderungs- und -schutzverträge (IFV) der Bundesrepublik Deutschland. https://www.bmwi.de/Navigation/DE/Service/Investitionsschutzvertraege/investitionsschutzvertraege.html. Accessed 20 Dec 2018

Brown C (2003) The Evolution and Application of Rules Concerning Independence of the International Judiciary. Law and Practice of Int'I Courts and Tribunals 2(1), 63-96.

Chesterman S (2008) An International Rule of Law? The American Journal of Comparative Law 56(2), 331-362.

Cohen HG, Follesdal A, Grossman N, Ulfstein G (2018) Legitimacy and International Courts - a Framework. In: Cohen HG et al., eds., Legitimacy and International Courts. Cambridge University Press, Cambridge.

Deutscher Richterbund (2016) Stellungnahme zur Errichtung eines Investitionsgerichts für TTIP - Vorschlag der Europäischen Kommission vom 16.09.2015 und 12.11.2015. https://www.bmwi.de/Redaktion/DE/Downloads/S-T/ stellungnahme-deutscher-richterbund-zur-errichtungeines-investitionsgerichts-fuer-ttip.pdf. Accessed $20 \mathrm{Dec}$ 2018.

European Commission (2017) Factsheet on Commission Proposal for a Multilateral Investment Court. http:// trade.ec.europa.eu/doclib/docs/2017/september/tradoc_156042.pdf. Accessed 20 Dec 2018

Grossman N (2009) Legitimacy and International Adjudicative Bodies. George Washington Int'I Law Review 41, 107.

Helfer LR, Slaughter AM (2005) Why States Create International Tribunals: a Response to Professors Posner and Yoo. California Law Review 93(3), 899-956.

Kaufmann-Kohler G, Potestà M (2016) Can the Mauritius Convention Serve as a Model for the Reform of InvestorState Arbitration in Connection with the Introduction of a Permanent Investment Tribunal or an Appeal Mechanism? CIDS Working Paper, Geneva. http://www.uncitral.org/ pdf/english/commissionsessions/unc/unc-49/CIDS_Research_Paper_-_Can_the_Mauritius_Convention_serve as_a_model.pdf. Accessed 20 Dec 2018.

Kaufmann-Kohler G, Potestà M (2017) The Composition of a Multilateral Investment Court and of an Appeal Mechanism for Investment Awards. CIDS Supplemental Report, Geneva. http://www.uncitral.org/pdf/english/ workinggroups/wg_3/CIDS_Supplemental_Report.pdf. Accessed 20 Dec 2018

Kosař D (2015) Selecting Strasbourg Judges: A Critique. In: Bobek M, ed. Selecting Europe's Judges: A Critical Review of the Appointment Procedures to the European Courts. Oxford University Press, Oxford.

Langford M, Behn D, Hilleren Lie R (2017) The Revolving Door in International Investment Arbitration. Journal of Int'l Economic Law 20(2), 301-323.

Mahoney P (2008) The International Judiciary - Independence and Accountability. Law \& Practice of Int'l Courts and Tribunals 7, 313-349.

doi: $10.1163 / 157180309 \times 399654$

MacKenzie R et al. (2010) Selecting International Judges: Principle, Process, and Politics. Oxford University Press, Oxford.

Posner EA, Yoo JC (2005) Judicial Independence in International Tribunals.California Law Review 93(1), 1-27. doi: $10.15779 / Z 38070 P$

Steger D (2015) The Founding of the Appellate Body. In Marceau G, ed, A History of Lawyers in the GATT/WTO: The Development of the Rule of Law in the Multilateral Trading System. Cambridge University Press, Cambridge.

United Nations Commission on Trade and Development UNCTAD (2018a) Recent Developments in the International Investment Regime. IIA Issues Note, May 2018. http:// unctad.org/en/PublicationsLibrary/diaepcbinf2018d1_ en.pdf. Accessed 20 Dec 2018
United Nations Commission on Trade and Development UNCTAD (2018b) Investor-State Dispute Settlement: Review of Developments in 2017. IIA Issues Note, June 2018. http://unctad.org/en/PublicationsLibrary/diaepcbinf2018d2_en.pdf. Accessed 20 Dec 2018.

Van Harten G (2010) Perceived Bias in Investment Treaty Arbitration. In: Waibel M, Kaushal A et al, eds., The Backlash Against Investment Arbitration. Kluwer, Amsterdam.

Voeten E (2009) The Politics of International Judicial Appointments. Chicago Journal of Int'I Law 9(2), 387-405.

Voeten E (2013) International Judicial Independence. In: Dunoff JA, Pollack MA, eds. Interdisciplinary Perspectives on International Law and International Relations - the State of the Art. Cambridge University Press, Cambridge.

Voeten E (2014) International Judicial Behavior. In: Romano C, Alter KJ, Shany Y, eds., The Oxford Handbook of International Adjudication. Oxford University Press, Oxford.

Zuleta E (2015) The Challenges of Creating a Standing International Investment Court. In: Kalicki JE, Joubin-Bret A, eds., Reshaping the Investor-state Dispute Settlement System. Brill Nijhoff, Leiden.

\section{AUTOR}

Prof. Dr. Christian Hederer, LL.M.

Fachbereich Wirtschaft, Informatik, Recht Technische Hochschule Wildau

E-Mail für Korrespondenz:

christian.hederer@th-wildau.de

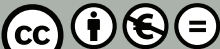

